\title{
Small-scale oscillations in a quiescent prominence observed by HINODE/SOT
}

\section{Prominence oscillations}

\author{
Z. Ning ${ }^{1}$, W. $\mathrm{Cao}^{2}$, T. J. Okamoto ${ }^{3}$, K. Ichimoto ${ }^{3,4}$, and Z. Q. Qu ${ }^{5}$ \\ 1 Purple Mountain Observatory, Nanjing 210008, PR China \\ e-mail: ningzongjun@pmo.ac.cn \\ ${ }^{2}$ Center for Solar Terrestrial Research, New Jersey Institute of Technology, Newark, NJ 07102, USA \\ e-mail: wcao@bbso.njit.edu \\ 3 National Astronomical Observatory of Japan, Mitaka, Tokyo 181-8588, Japan \\ e-mail: joten.okamoto@nao.ac.jp \\ 4 Kwasan and Hida Observatories, Kyoto University, Yamashina, Kyoto 607-8471, Japan \\ e-mail: ichimoto@kwasan.kyoto-u.ac.jp \\ 5 Yunnan Astronomy Observatory, Kunming 650011, PR China
}

Received 25 August 2008 / Accepted 6 March 2009

\section{ABSTRACT}

\begin{abstract}
Context. Investigations of the behavior of small-scale threads can provide an alternative approach to studying prominence dynamics and understanding its origin and nature.

Aims. The behavior of threads are analyzed in a quiescent prominence, including drifting and both the horizontally and vertically oscillating motions. These indicate waves in the solar prominence.

Methods. We used the $\mathrm{H} \alpha$ images at a setting wavelength of $+0.076 \AA$. A quiescent prominence was observed by HINODE/SOT on 2008 January 15 for about $3 \mathrm{~h}$ in total.

Results. Consistent with previous findings, prominences show numerous thread-like structures. Some threads clearly exhibit both vertically and horizontally oscillatory motions, while others are only drifting. Complicated cases show both drifting and oscillatory motions simultaneously. In the upper part of the prominence, the threads are oscillating independently of each other. We find that three threads oscillate with the same phase for at least two periods. The oscillations seem to be strongly damped since they disappear after a few periods. The maximum number of observed periods is 8 in our observations. In the lower part of the prominence, however, the different threads have a mixed character with the individual oscillatory motions unstable for one entire period. Most oscillatory motions will disappear after a half period or less, while the new oscillatory motions are excited nearby. A 5-min period is predominant, and the oscillating amplitudes show an average value of $\pm 3.5 \mathrm{~km} \mathrm{~s}^{-1}$. We find some upflows in the spicule layer, and they appear to transport the mass from photosphere (or spicules themselves) to the prominence. These upflows have an average velocity amplitude of $0.8 \mathrm{~km} \mathrm{~s}^{-1}$.

Conclusions. The threads exhibit three distinct behaviors. The first is only drifting, the second is typically oscillating, and the third shows both characteristics. There are no substantial differences between the periods of horizontally and vertically oscillating threads in this prominence.
\end{abstract}

Key words. Sun: corona - Sun: prominences - Sun: oscillations

\section{Introduction}

Previous observations have pointed out the existence of two different types of prominence oscillations (Oliver \& Ballester 2002): large-amplitude oscillations (amplitude $\sim 20 \mathrm{~km} \mathrm{~s}^{-1}$ ) and small-amplitude oscillations (amplitude $\leq 2 \sim 3 \mathrm{~km} \mathrm{~s}^{-1}$ ). The former are flare-induced oscillations that affect the whole prominence, while the latter are quiescent prominence oscillations that seem to be local in nature. It has been suggested that the driver of the large-amplitude oscillations was either a wave caused by a solar flare or nearby activities. Both of them disturb the prominence and induce oscillations. From observations, such a wave was identified as Moreton or EIT waves (e.g. Ramsey \& Smith 1966; Eto et al. 2002; Okamoto et al. 2004), and these activities were either microflares (Jing et al. 2003), emerging flux (Isobe \& Tripathi 2006; Isobe et al. 2007; Xu et al. 2008), or surges
(Chen et al. 2008). Observational studies of quiescent prominences have revealed a great diversity in characteristic periods ranging from less than $1 \mathrm{~min}$ up to $90 \mathrm{~min}$ (Bashkirtsev \& Mashinich 1984; Tsubaki \& Takeuchi 1986; Tsubaki et al. 1987a,b, 1988; Molowny-Horas et al. 1999; Terradas et al. 2002). These oscillation periods are typically grouped into four categories: very short $(\leq 1 \mathrm{~min})$, short $(1-20 \mathrm{~min})$, intermediate (20-40 min), and long ( $\geq 40 \mathrm{~min}$ ) (e.g., Engvold 2001). Understanding the origin and nature of these oscillations is one of the most important issues in current prominence seismology.

Observations reveal that solar prominences are sheets of relatively cool and dense plasma embedded in the surrounding hotter corona, and they have two linked categories of structures: "spines" and "barbs" (e.g. Engvold 1998; Zirker et al. 1998; Zong et al. 2003; Lin et al. 2003). A "spine" is the horizontal axis of the prominence, while "barbs" connect to the high spine 
and terminate in the chromosphere. High-resolution $\mathrm{H} \alpha$ filtergrams from ground-based observations have resolved numerous very thin, thread-like structures (fibrils) in prominence spines (Lin et al. 2003, 2005a,b, 2007). The widths of the threads are typically between 0.2 and 0.3 arcsec. The barbs are sheets of mass, composed of parallel narrow threads similar to those in the spine. Two-dimensional high-resolution observations of prominences have provided interesting information about the thread oscillations, which are identified as small-amplitude waves propagating along a number of threads, with an average velocity of $12 \mathrm{~km} \mathrm{~s}^{-1}$ and a wavelength of about 4 arcsec. Detailed studies show that individual threads or groups of threads may oscillate independently with their own periods and phases (Yi \& Engvold 1991; Yi et al. 1991). Observations from space confirm these findings, and reveal additional dynamics related to the coronal structure (Schrijver et al. 1999; Karpen et al. 2006; Okamoto et al. 2007). Using the HINODE/SOT (Ichimoto et al. 2004, 2008; Kosugi et al. 2007; Tsuneta et al. 2008; Suematsu et al. 2008; Shimizu et al. 2008) data, Okamoto et al. (2007) have found that the threads in a solar prominence underwent vertically (i.e. in the plane of the sky on the limb) oscillating motions with a typical period between $\sim 240$ and 250 s. One thread was discovered to be oscillating along its entire length, which was as long as $16000 \mathrm{~km}$. Lin et al. (2005b, 2007) studied filaments on the disk to find that the detected oscillations (in Doppler velocity) probably correspond to transverse oscillations of fine structures.

Until now, studies of small-amplitude oscillations in prominences have been made in the vertical plane of the solar surface (e.g. Lin et al. 2005a,b, 2007; Okamoto et al. 2007), while horizontally oscillating motions parallel to solar surface are poorly known, although large-scale transverse oscillations in quiescent prominences have been reported (Berger et al. 2008). In contrast to the Okamoto et al. (2007) data set, which observed a prominence along the spine axis, we used different HINODE/SOT data, in which a limb prominence was observed perpendicular to its axis. This gives us a chance to study prominence oscillations parallel and perpendicular to the solar surface.

\section{Observations and measurements}

The SOT (Solar Optical Telescope) aboard HINODE obtains diffraction-limited images with a $50-\mathrm{cm}$ aperture telescope from a Sun-synchronous orbit. The SOT provides us with an opportunity to study small-scale dynamics in prominence thanks to high image stability and high spatial resolution. In our data set, the prominence images were taken by the Narrowband Filter Imager (NFI) at a wavelength of $\mathrm{H} \alpha+0.076 \AA$ (which is very close to $\mathrm{H} \alpha$ line center), $\mathrm{H} \alpha-0.340 \AA$ (we actually tried to observe at $\mathrm{H} \alpha \pm 0.21 \AA$ after taking the possible offset into account), and the CaII H-line (3968 $\AA$ ). We show the results from the analysis of the $\mathrm{H} \alpha$ images at $+0.076 \AA$ in this paper. The spatial resolution of the NFI (or $\mathrm{H} \alpha$ ) is 0.16 arcsec per pixel after $2 \times$ 2 binning (to have a larger field of view and signal/noise ratio). The time cadence of the data set is $30 \mathrm{~s}$.

The prominence studied here was located on the solar west limb, and it was observed by HINODE/SOT from 10:33:18 to 13:28:22 UT on 2008 January 15. The spines of this prominence were almost parallel to the solar equator, thus our observations were perpendicular to its spine axis. Figure 1 shows the $\mathrm{H} \alpha+0.076 \AA$ image at 12:12:17 UT. Spicules were also seen above solar surface up to $5000 \mathrm{~km}$. The prominence had two spines indicated as "SP1" and "SP2" in Fig. 1. SP1 has a large cross section of $\sim 180 \times 10^{6} \mathrm{~km}^{2}$, and could hold most of the mass of the prominence. It reaches a maximum height at $16000 \mathrm{~km}$ above the solar limb, while SP2 has a section of $\sim 7 \times$ $10^{6} \mathrm{~km}^{2}$ and a maximum height of $18000 \mathrm{~km}$. On the other hand, SP1 had barbs embedded in spicules, while SP2 was suspended in the atmosphere in the time window of our observations. To investigate oscillatory behaviors in the vertical and horizontal directions, we used six slits to get time slices in each direction, such as solids lines marked by from $1 y$ to $6 y$, or $1 x$ to $6 x$ in Fig. 1. These slits work simultaneously. Thus, we could detect the oscillatory behavior at the same position, but in different directions. All images were aligned using the solar limb prior to making the time slices.

Figure 2 shows the space-time slices of intensities along the vertical slits, i.e. perpendicular to the solar surface, from $1 y$ to $6 y$, respectively. The spine SP1 is easily seen in the slit $4 y$. The prominence shows its barb's connection between the spine (SP1) and the photosphere. Consistent with previous findings (Lin et al. 2005a,b, 2007), the prominence spines, SP1 and SP2, are composed of numerous thin threads. Different threads are located at different heights. This fact can be confirmed by the intensity variation along the slit. The prominence threads exhibit vertically oscillating motions, although they are faint and some are difficult to distinguish from the evolution of intensity. To clearly see these threads and their behaviors, we calculated the intensity-gradient along the slit, i.e., from bottom (close the solar surface) to top. Figure 2 also shows the time evolution of the gradient diagram, in which the threads and their oscillations are apparent than before. Thus, one thread is divided into two bands, the white one indicates the thread boundary where the intensity increases along slits, and the nearby black band is the other boundary where the intensity decreases. In these figures, the white bands are always beneath the black ones. We find most of the prominence threads are vertically oscillating with few exceptions. Meanwhile, some threads are drifting upward or downward.

Using the same method, Fig. 3 shows the time evolution of the intensities and their gradients at the horizontal slits from $1 x$ to $6 x$. The gradient is computed along the solar $Y$ direction, i.e. from north to south. The prominence exhibits horizontally oscillating motions, especially at the spine of SP2, i.e., at slits $4 x-6 x$. The SP1 spine has a large width, as wide as $10 \operatorname{arcsec}(\sim 7500 \mathrm{~km})$, as seen at slits $1 x-3 x$. We find that some, but not all, threads are oscillating horizontally (see details later). And some threads are drifting southward or northward (upward or downward in Fig. 3).

\section{Results}

To measure the parameters of individual oscillations, Figs. 4 and 5 show the horizontally and vertically oscillating motions in detail. Thirteen individual threads are identified, as marked in Fig. 4. Ten of them show horizontally oscillating motions, while the other 3 are only drifting. At slit $3 x$ in Fig. 4B1, the prominence spine SP1 shows its edges with horizontally oscillating motions, such as G1, G2, G4, G5, and G7. It is possible that $\mathrm{G} 1, \mathrm{G} 4$, and G5 can be considered as the oscillating motion in a thread, because they are located on the top edge of SP1. In the middle of SP1, some threads also show horizontally oscillating motions, i.e. G3 and G6. At the top slit $6 x$ in Fig. 4B2, three groups of oscillatory motions are marked, for instance G8, G12, and G13. G12 has three threads, which are horizontally oscillating with the same phase, at least lasting for two periods. Then two threads disappear, while the other drifts northward with a speed of $8.1 \mathrm{~km} \mathrm{~s}^{-1}$. Its oscillations disappear completely after 


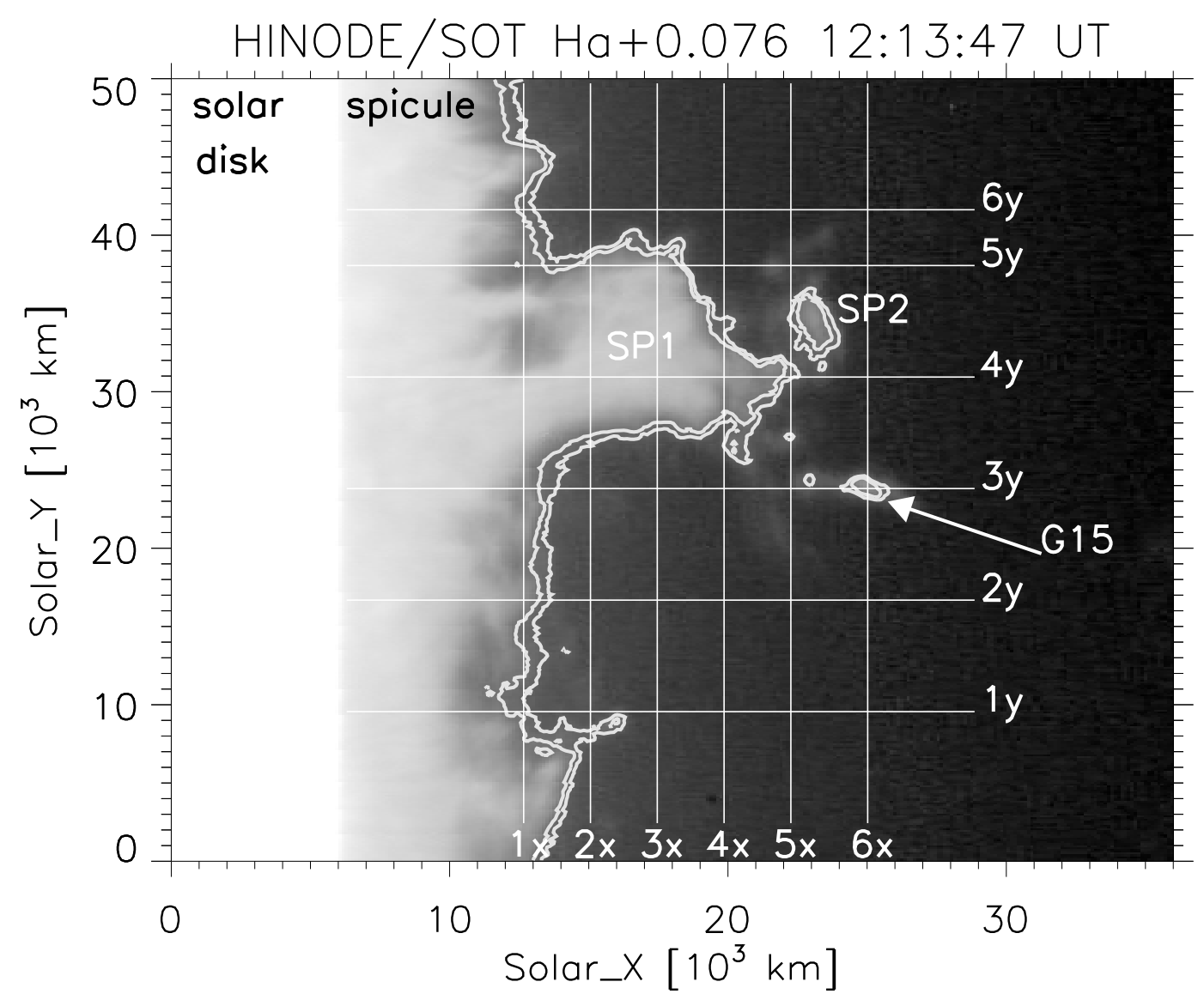

Fig. 1. HINODE/SOT H $\alpha$ images (at the setting wavelength of $+0.076 \AA$ ) of the quiescent prominence on the solar west limb on 2008 January 15. Above the limb, ubiquitous vertical spicules are seen. The prominence has two spines marked by "SP1" and "SP2". The solid lines indicate the horizontal and vertical slits, and their time slices are shown in Figs. 2-5. The arrow points to the oscillating thread of "G15" shown in Figs. 4 and 5. The intensity of the prominence in $\mathrm{H} \alpha+0.076 \AA$ radiation is about $1 \%$ of that of the on-disk photosphere. The contour levels are 0.5 and 0.55 of the maximum value. Solar south is up and west is right.

two periods. This fact implies that the prominence oscillations are strongly damped, which is consistent with previous observations of damped oscillations (e.g. Malville \& Schindler 1981; Terradas et al. 2001; Arregui et al. 2008). The spatial width of these three threads is $\sim 9 \operatorname{arcsec}(6800 \mathrm{~km})$. Thread G13 drifts southward at the beginning, with a speed of $4.8 \mathrm{~km} \mathrm{~s}^{-1}$. After about $50 \mathrm{~min}$, it starts its horizontally oscillating motions, which show an average period of $\sim 290 \mathrm{~s}$. G9, G10, and G11 are identified as the threads that are only drifting northward or southward. Their drifting speeds are estimated as $9.2,7.5$, and $3.2 \mathrm{~km} \mathrm{~s}^{-1}$, respectively.

Meanwhile, three threads are identified in the slit $3 y$ in Fig. 5A1, i.e. G14-G16. These three threads oscillate with an average period of $330 \mathrm{~s}$, which is a little longer than that of horizontally oscillating motions. Both G14 and G15 threads are drifting upward simultaneously. Several threads drift upward as quickly as $24 \mathrm{~km} \mathrm{~s}^{-1}$ at slit $4 y$ in Fig. 2. We find that the oscillating threads are drifting downward, especially at slit $2 y$ in Fig. 2, with a velocity of $2.3 \mathrm{~km} \mathrm{~s}^{-1}$. By comparing Figs. 4B2 with $5 \mathrm{~A} 1$, we find that the G15 thread only shows vertically oscillating motions, no horizontally oscillating motions. From Fig. 1, we roughly estimate the cross-sectional area of thread G15 is $\sim 1 \operatorname{arcsec}^{2}\left(\sim 560000 \mathrm{~km}^{2}\right)$. Figure 5A2 shows a complicated case of thread oscillation. It is difficult to identify an individual thread or an individual oscillating motion independently. Some oscillating threads are mixed with other threads, and they oscillate with different phases. Thus, an oscillating motion seems to disappear, while another new oscillation is excited nearby simultaneously. Probably, these appearing and/or disappearing behaviors are due to oscillations into or out of the slit surfaces here. Such complicated cases are also seen in other vertical slits, especially at the slits $3 y-5 y$ in Fig. 2.

We measured the parameters, including the periods, amplitudes, and oscillating velocities, of 16 individual threads that are clearly identified here, as listed in Table 1, and the drifting velocities are also given. The velocities and amplitudes are measured on the slit plane.

In Fig. 2, we find some upflows in the spicule layer from the photosphere to the prominence, as indicated by the white arrows at slits $1 y, 3 y$, and $5 y$. They show an average velocity of $0.8 \mathrm{~km} \mathrm{~s}^{-1}$. These drifting motions with no evidence of oscillation seem to be mass motions.

\section{Conclusions and discussions}

High-resolution observations from SOT/HINODE reveal numerous small-scale threads in a quiescent prominence. This is consistent with the previous studies of filament close to disk center (Lin et al. 2003, 2005a,b, 2007). This evidence seems to favor a fibril approach to prominence modeling (e.g. Joarder et al. 1997; Díaz et al. 2001; 2005; Díaz \& Roberts 2006). We find three different behaviors of small-scale thread motions in the prominence. The first is only drifting, and the second is typically oscillating, in the horizontal and/or vertical planes, and the third 


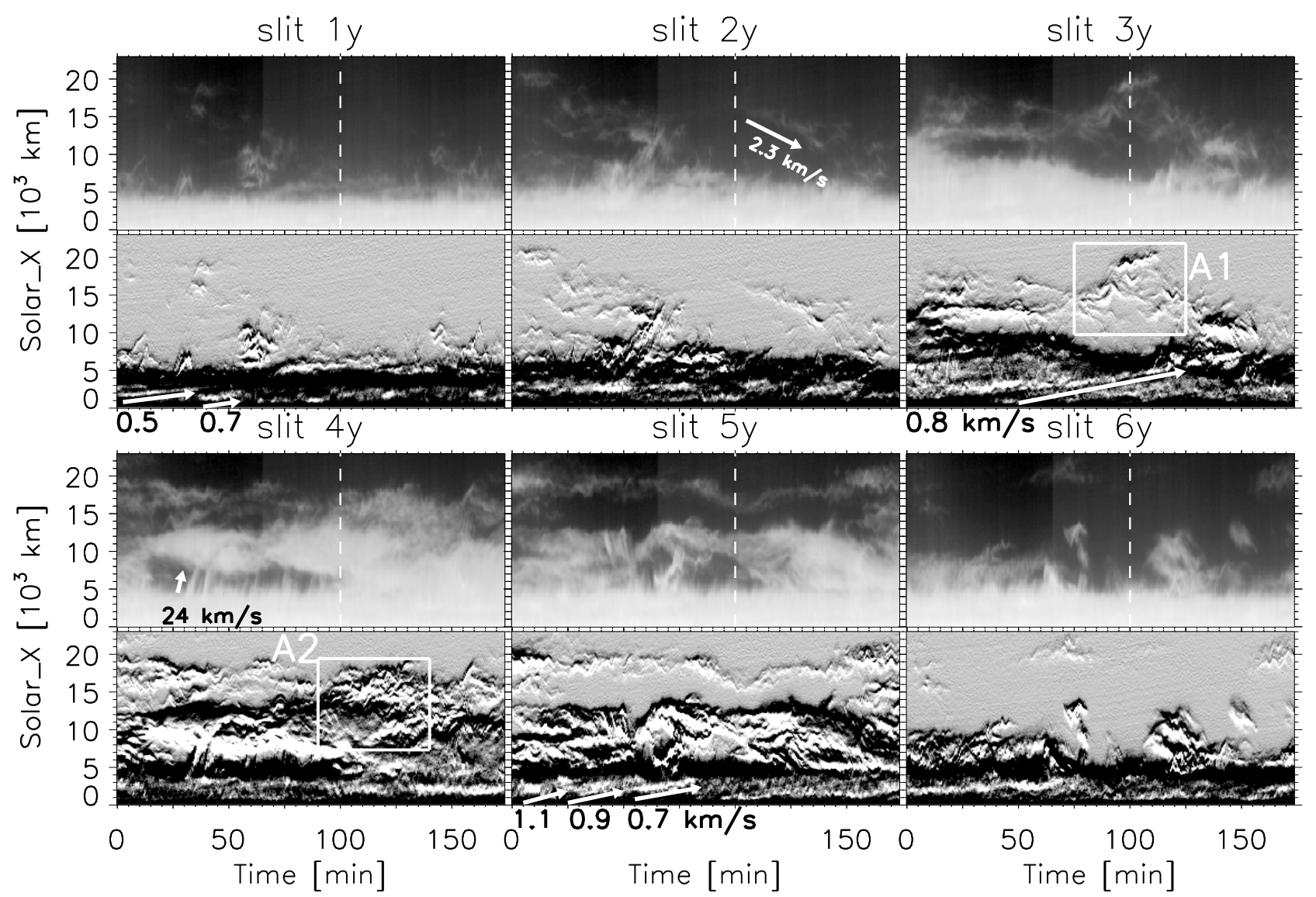

Fig. 2. Space-time slices along the vertical slits of $1 y-6 y$ in Fig. 1. Both intensity and its gradient along solar $X$ axis from bottom (close the solar surface) to top at each slit are shown. The two-dimensional image at the time indicated by the dashed line is shown in Fig. 1. Six upflows are identified at the slits $1 y, 3 y$, and $5 y$. White arrows at slits $2 y$ and $4 y$ point out the downward and upward drifting structures, respectively (see text for details). The numbers show the speed values. The space-derivative in the boxes of "A1" and "A2" is shown in Fig. 5.

shows both characteristics. These facts should be accounted by future modeling of prominence. The three different behaviors of the small-scale threads would be an observational constraint on the mechanism of oscillation in prominences.

Investigation of the behavior of threads can give us important information on prominence dynamics. Our observations show that the small-scale threads in prominences (spine or barb) are highly dynamic. The oscillating motions are worthy of study because they contain rich information on the physics of prominences. In the prominence studied here, the threads exhibit oscillating motion, whether in the horizontal or vertical planes (with respect to the solar surface). We find oscillation periods ranging from 210 to $525 \mathrm{~s}$ (typical value of $\sim 300 \mathrm{~s}$ ), the peak-to-peak amplitude between 720 and $1440 \mathrm{~km}$ (average value of $\sim 1000 \mathrm{~km}$ ), and the oscillating velocity between 5.0 and $9.1 \mathrm{~km} \mathrm{~s}^{-1}$ (with an average value of $\sim 7.0 \mathrm{~km} \mathrm{~s}^{-1}$ or amplitude of $\pm 3.5 \mathrm{~km} \mathrm{~s}^{-1}$ ). These typical parameters are consistent with the previous finding using a Ca II K line in a quiescent prominence (e.g. Tsubaki \& Takeuchi 1986; Tsubaki et al. 1987a,b, 1988). We do not find any fundamental difference between the periods of the horizontally and vertically oscillating threads. The oscillations seem to be strongly damped since they disappear after a few periods. The maximum number of observed periods is 8 in our observations, as listed in Table 1. Another typical behavior of the thread's motion is discovered as drifting in the horizontal and vertical planes. We do not find any difference between the horizontally and vertically drifting threads, which show an average drifting speed of $4.6 \mathrm{~km} \mathrm{~s}^{-1}$. Such drifting motions can be caused by the rearrangement of magnetic field lines carrying mass. The complicated case is that some threads exhibit both the drifting and oscillating motions simultaneously. We also find slow upflows in the spicule layer underlying the prominence, which could transport the mass from the photosphere to the prominence.

We do not find that individual oscillations change their period (increasing or decreasing) with time. In the upper part of prominence, each individual thread oscillate independently, while the different threads are mixed in the lower layer and oscillate with various phases. From the observations, an oscillating behavior only lasts for one or half a period; however, new one appears nearby simultaneously. This indicates that the exciters or drivers of such oscillations are numerous and small scale. The origin of the prominence oscillations is still unknown. The Sun was quiet, and no active regions were identified on the solar disk around 2008 January 15 . The exciter of prominence oscillations does not seem to be related to flares or other activity that was reported before (e.g. Jing et al. 2003; Okamoto et al. 2004; Isobe \& Tripathi 2006; Isobe et al. 2007; Xu et al. 2008; Chen et al. 2008). It is generally thought that the thread oscillations stem from magnetoacoustic waves (e.g. Joarder et al. 1997; Díaz et al. 2005), which are possibly transported from the photospheric regions where the magnetic field lines of prominence are rooted.

An open question is why 5-min period oscillations dominate in this prominence, while Okamoto et al. (2007) find a typical period of $\sim 240$ to $250 \mathrm{~s}$ in another prominence. It is reminiscent of 3- and 5-min oscillations in the solar atmosphere. If the oscillations are excited in the photospheric regions, the 



Fig. 3. As in Fig. 2, but space-time slices along the horizontal slits of $1 x-6 x$ in Fig. 1. Both intensity and its space-derivative (along solar $Y$ axis from bottom (north) to top (south)) at each slit are shown. The space-derivative in the boxes of "B1" and "B2" are shown in Fig. 4.

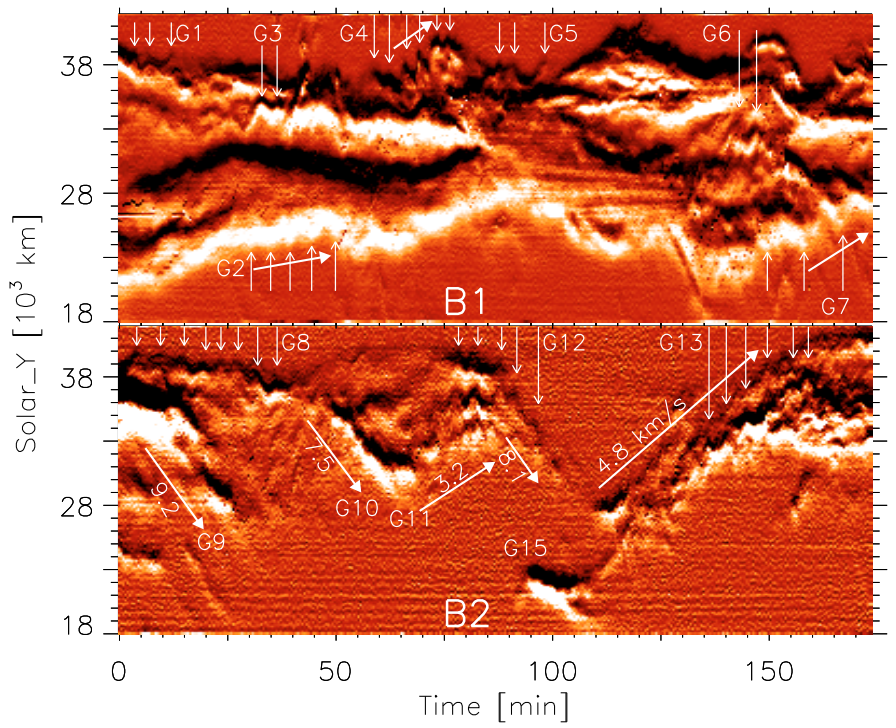

Fig. 4. Space-time slices of space-derivative at B1 and B2 (in Fig. 3). The thread's oscillating and drifting motions are marked by arrows (see text in details). The numbers show the speed values.

prominence should terminate their spine feet in the network regions. Observations show that 3-min oscillations are related to the internetwork regions, while the 5-min oscillations dominate in the network regions (e.g. Lites et al. 1993; Carlsson et al. 1997; Judge et al. 1997; Wikstl et al. 2000; Hansteen et al. 2000; Banerjee et al. 2001; Muglach 2003; McAteer et al. 2004). It is

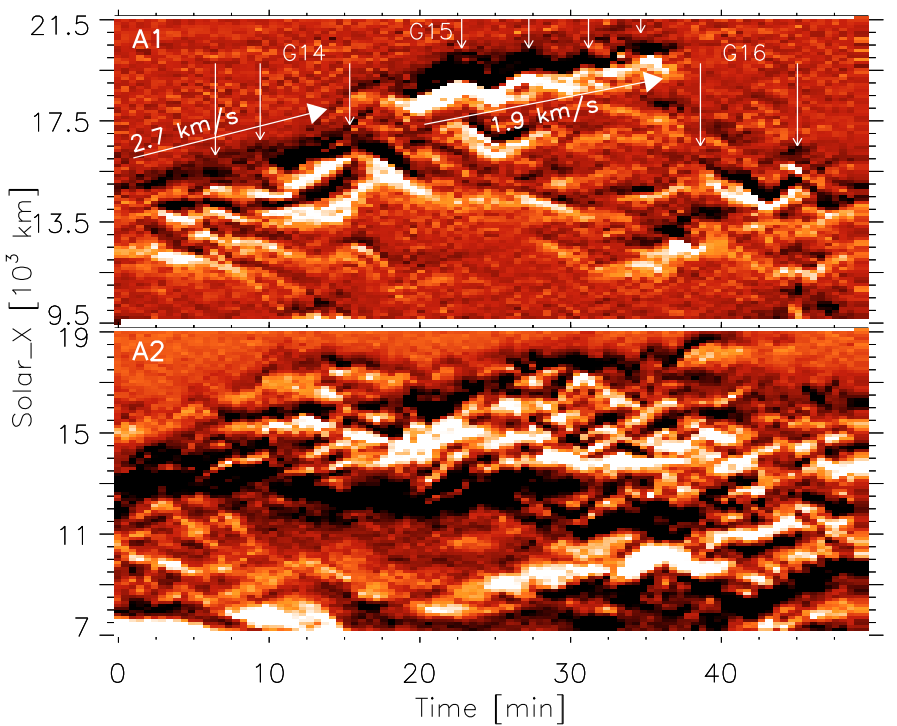

Fig. 5. As in Fig. 4, space-time slices of space-derivative at A1 and A2 in Fig. 2 (see text for details).

possible that the prominence in Okamoto et al. (2007) embeds its feet in both the internetworks and the networks, while the prominence studied here roots only in the network. If this case is true, it is expected to find that the oscillation periods dominate $3 \mathrm{~min}$ in a prominence that terminates its feet in the internetwork regime. Another possibility is that the prominence oscillation period is determined by the intrinsic physics. For example, it 
Table 1. Parameters of thread oscillating motions in Figs. 4 and 5.

\begin{tabular}{clllll}
\hline \hline & $\begin{array}{l}\text { Number of } \\
\text { observed } \\
\text { periods }\end{array}$ & Period & $\begin{array}{l}\text { Oscillation } \\
\text { amplitude } \\
(\mathrm{km})\end{array}$ & $\begin{array}{l}\text { Phase } \\
\text { velocity } \\
\left(\mathrm{km} \mathrm{s}^{-1}\right)\end{array}$ & $\begin{array}{l}\text { Drifting } \\
\text { velocity } \\
\left(\mathrm{km} \mathrm{s}^{-1}\right)\end{array}$ \\
\hline G1 & 3 & 255 & 1080 & 8.5 & - \\
G2 & 5 & 292 & 960 & 6.6 & 1.0 \\
G3 & 3 & 210 & 720 & 6.9 & - \\
G4 & 6 & 210 & 960 & 9.1 & 4.0 \\
G5 & 3 & 315 & 840 & 5.3 & - \\
G6 & 2 & 240 & 1080 & 9.0 & - \\
G7 & 3 & 525 & 1320 & 5.0 & 3.6 \\
G8 & 8 & 278 & 860 & 6.9 & - \\
G9 & - & - & - & - & 9.2 \\
G10 & - & - & - & - & 7.5 \\
G11 & - & - & - & - & 3.2 \\
G12 & 5 & 278 & 1080 & 7.8 & 8.1 \\
G13 & 6 & 276 & 840 & 6.1 & 4.8 \\
G14 & 3 & 360 & 1320 & 7.3 & 2.7 \\
G15 & 4 & 240 & 1080 & 9.0 & 1.9 \\
G16 & 2 & 390 & 1440 & 7.4 & - \\
\hline
\end{tabular}

could be more closely related to the resonance frequency determined by the magnetic filed configurations. Because the network (5-min) oscillations can explain how the vertical oscillations are driven in the prominence, but it is difficult for them to drive horizontally oscillating motions. The 5-min oscillation is a radial oscillation in the solar atmosphere.

The primary results of this paper were derived from the data set of 2008 January 15. A joint analysis of all data, including those from the blue wing $(-0.340 \AA)$ of $\mathrm{H} \alpha$ and $\mathrm{Ca} \mathrm{H}$-line observations, which probably give information coming from or near the prominence-corona transition region, will be done in the near future.

Acknowledgements. We would like to thank the anonymous referee for the valuable comments to improve this manuscript. HINODE is a Japanese mission developed and launched by ISAS/JAXA, with NAOJ as domestic partner and NASA and STFC (UK) as international partners. It is operated by these agencies in co-operation with ESA and NSC (Norway). Z. Ning and $\mathrm{Z}$. Qu received financial accommodation from NAOJ and ISAS/JAXA for two months. W. Cao gratefully acknowledges the support of the NSF through ATM-0847126, ATM-0745744, and NASA through NASA-NNX08BA22G. This work is also supported by NSF of China under grants 10603014, 10843005, 10833007, 973 Program under grant 2006CB806302, and CAS Project under grant KJCX2-YW-T04.

\section{References}

Arregui, I., Terradas, J., Oliver, R., \& Ballester, J. L. 2008, ApJ, 682, L141 Banerjee, D., O’Shea, E., Doyle, J. G., \& Goossens, M. 2001, A\&A, 371, 1137 Bashkirtsev, V. S., \& Mashnich, G. P. 1984, Sol. Phys., 91, 93

Berger, T. E., Shine, R. A., Slater, G. L., et al. 2008, ApJ, 676, L89 Carlsson, M., Judge, P. G., \& Wilhelm, K. 1997, ApJ, 486, L63 Chen, P. F., Innes, D. E., \& Solanki, S. K. 2008, A\&A, 484, 487

Díaz, A. J., \& Roberts, B. 2006, A\&A, 458, 975

Díaz, A. J., Oliver, R., Erdélyi, R., \& Ballester, J. L. 2001, A\&A, 379, 1083

Díaz, A. J., Oliver, R., \& Ballester, J. L. 2005, A\&A, 440, 1167

Engvold, O. 1998, IAU Colloq., 167: New Perspectives on Solar Prominences, 150,23

Engvold, O., Jakobsson, H., Tandberg-Hanssen, E., Gurman, J. B., \& Moses, D. 2001, Sol. Phys., 202, 293

Eto, S., Isobe, H., Narukage, N., et al. 2002, PASJ, 54, 481

Hansteen, V. H., Betta, R., \& Carlsson, M. 2000, A\&A, 360, 742

Jing, J., Lee, J., Spirock, T. J., et al. 2003, ApJ, 584, L103

Judge, P., Carlsson, M., \& Wilhelm, K. 1997, ApJ, 490, L195

Lin, Y., Engvold, O. R., \& Wiik, J. E. 2003, Sol. Phys., 216, 109

Lin, Y., Engvold, O., Rouppe van der Voort, L., Wiik, J. E., \& Berger, T. E. 2005a, Sol. Phys., 226, 239

Lin, Y., Wiik, J. E., Engvold, O., Rouppe van der Voort, L., \& Frank, Z. A. 2005b, Sol. Phys., 227, 283

Lin, Y., Engvold, O., Rouppe van der Voort, L. H. M., \& van Noort, M. 2007, Sol. Phys., 246, 65

Lites, B. W., Rutten, R. J., \& Kalkofen, W. 1993, ApJ, 414, 345

Ichimoto, K., Tsuneta, S., Suemastsu, Y., et al. 2004, SPIE, 5487, 1142

Ichimoto, K., Lites, B., Elmore, D., et al. 2008, Sol. Phys., 249, 233

Isobe, H., \& Tripathi, D. 2006, A\&A, 449, L17

Isobe, H., Tripathi, D., Asai, A., \& Jain, R. 2007, Sol. Phys., 246, 89

Joarder, P. S., Nakariakov, V. M., \& Roberts, B. 1997, Sol. Phys., 173, 81

Karpen, J. T., Antiochos, S. K., \& Klimchuk, J. A. 2006, ApJ, 637, 531

Kosugi, T., Matsuzaki, K., Sakao, T., et al. 2007, Sol. Phys., 243, 3

Malville, J. M., \& Schindler, M. 1981, Sol. Phys., 70, 115

McAteer, R. T. J., Gallagher, P. T., Bloomfield, D. S., et al. 2004, ApJ, 602, 436

Molowny-Horas, R., Wiehr, E., Balthasar, H., Oliver, R., \& Ballester, J. L. 1999, JOSO Annu. Rep., 1998, 126, 126

Muglach, K. 2003, A\&A, 401, 685

Oliver, R., \& Ballester, J. L. 2002, Sol. Phys., 206, 45

Okamoto, T. J., Nakai, H., Keiyama, A., et al. 2004, ApJ, 608, 1124

Okamoto, T. J., Tsuneta, S., Berger, T. E., et al. 2007, Science, 318, 1577

Ramsey, H. E., \& Smith, S. F. 1966, AJ, 71, 197

Schrijver, C. J., Title, A., Berger, T., et al. 1999, SoPh, 187, 261

Shimizu, T., Nagata, S., Tsuneta, S., et al. 2008, Sol. Phys., 249, 221

Suematsu, Y., Tsuneta, S., Ichimoto, K., et al. 2008, Sol. Phys., 249, 197

Terradas, J., Oliver, R., \& Ballester, J. L. 2001, A\&A, 378, 635

Terradas, J., Carbonell, M., Oliver, R., \& Ballester, J. L. 2005, A\&A, 434, 741

Tsubaki, T., \& Takeuchi, A. 1986, Sol. Phys., 104, 313

Tsubaki, T., Ohnishi, Y., \& Suematsu, Y. 1987a, PASJ, 39, 179

Tsubaki, T., Ohnishi, Y., \& Suematsu, Y. 1987b, PASJ, 39, 179

Tsubaki, T., Toyoda, M., Suematsu, Y., \& Gamboa, G. A. R. 1988, PASJ, 40, 121

Tsuneta, S., Ichimoto, K., Katsukawa, Y., et al. 2008, Sol. Phys., 249, 167

Wikstøl, Ø., Hansteen, V. H., Carlsson, M., \& Judge, P. G. 2000, ApJ, 531, 1150

Xu, X.-Y., Fang, C., \& Chen, P.-F. 2008, ChA\&A, 32, 56

Yi, Z., \& Engvold, O. 1991, Sol. Phys., 134, 275

Yi, Z., Engvold, O., \& Keil, S. L. 1991, Sol. Phys., 132, 63

Zirker, J. B., Engvold, O., \& Martin, S. F. 1998, Nature, 396, 440

Zong, W. G., Tang, Y. H., Fang, C., et al. 2003, A\&A, 412, 267 